\title{
The Wiener Polynomial of a Graph
}

\author{
Bruce E. Sagan ${ }^{1}$ \\ Department of Mathematics, Michigan State University, East Lansing, MI 48824-1027, U.S.A. \\ Yeong-Nan Yeh ${ }^{2}$ \\ Institute of Mathematics, Academia Sinica, Nankang, Taipei, Taiwan 11529, R.O.C. \\ and \\ Ping Zhang \\ Department of Mathematics, University of Texas, El Paso, TX 79968-0514, U.S.A.
}

April 9, 2003

Key Words: Wiener index, Wiener polynomial, distance, tree, unimodality

AMS subject classification (1991): Primary 05C12; Secondary 05A15, 05A20, 05C05.

${ }^{1}$ Supported in part by grant NSC 82-0501-1-001-A2 from the National Science Council, Taiwan, R.O.C.

${ }^{2}$ Supported in part by grant NSC-83-0208-M001-68 from the National Science Council, Taiwan, R.O.C. 
Proposed running head:

The Wiener polynomial

Send proofs to:

Bruce E. Sagan

Department of Mathematics

Michigan State University

East Lansing, MI 48824-1027

U.S.A.

FAX: 517-432-1562

e-mail: sagan@math.msu.edu 


\begin{abstract}
The Wiener index is a graphical invariant that has found extensive application in chemistry. We define a generating function, which we call the Wiener polynomial, whose derivative is a $q$-analog of the Wiener index. We study some of the elementary properties of this polynomial and compute it for some common graphs. We then find a formula for the Wiener polynomial of a dendrimer, a certain highly regular tree of interest to chemists, and show that it is unimodal. Finally, we point out a connection with the Poincaré polynomial of a finite Coxeter group.
\end{abstract}




\section{$1 \quad$ Introduction and elementary properties}

Let $d(u, v)$ denote the distance between vertices $u$ and $v$ in a graph $G$. Throughout this paper we will assume that $G$ is connected. The Wiener index of $G$ is defined as

$$
W(G)=\sum_{\{u, v\}} d(u, v)
$$

where the sum is over all unordered pairs $\{u, v\}$ of distinct vertices in $G$. The Wiener index was first proposed by Harold Wiener [12] as an aid to determining the boiling point of paraffin. Since then, the index has been shown to correlate with a host of other properties of molecules (viewed as graphs). For more information about the Wiener index in chemistry and mathematics see [5] and [2], respectively.

We wish to define and study a related generating function. If $q$ is a parameter, then the Wiener polynomial of $G$ is

$$
W(G ; q)=\sum_{\{u, v\}} q^{d(u, v)}
$$

where the sum is taken over the same set of pairs as before. It is easy to see that the derivative of $W(G ; q)$ is a $q$-analog of $W(G)$ (see Theorem 1.1, number 5). In the rest of this section we will derive some basic properties of $W(G ; q)$ and find its value when $G$ specializes to a number of simple graphs. In Section 2 we will compute the Wiener polynomial of a dendrimer $D_{n, d}$, a certain type of highly regular tree that models various chemical molecules. This permits us to rederive results of Gutman and his coauthors [6]. We then use this formula to show that the coefficients of $W\left(D_{n, d} ; q\right)$ are unimodal. Finally we end with a section on comments and open questions. In particular, we point out the connection with the Poincaré polynomial of a Coxeter group.

In what follows, any terms that are not defined will be found described in the text of Chartrand and Lesniak [3]. We will use $|S|$ to denote the cardinality of a set $S$. Also, if $f(q)$ is a polynomial in $q$ then $\operatorname{deg} f(q)$ is its degree and $\left[q^{i}\right] f(q)$ is the coefficient of $q^{i}$. The next theorem summarizes some of the properties of $W(G ; q)$. Its proof follows easily from the definitions and so is omitted.

Theorem 1.1 The Wiener polynomial satisfies the following conditions.

1. $\operatorname{deg} W(G ; q)$ equals the diameter of $G$.

2. $\left[q^{0}\right] W(G ; q)=0$.

3. $\left[q^{1}\right] W(G ; q)=|E(G)|$ where $E(G)$ is the edge set of $G$. 
4. $W(G ; 1)=\left(\begin{array}{c}|V(G)| \\ 2\end{array}\right)$ where $V(G)$ is the vertex set of $G$.

5. $W^{\prime}(G ; 1)=W(G)$

We will next find the Wiener polynomial of some specific graphs. We let $K_{n}, P_{n}, C_{n}$ and $W_{n}$ denote the complete graph, path, cycle and wheel on $n$ vertices, respectively. Also let $Q_{n}$ be the cube of dimension $n$ and $K_{m, n}$ be the complete bipartite graph on parts of size $m$ and $n$. Finally, $P$ denotes the Petersen graph. Determining the Wiener polynomials of these graphs is a matter of simple counting, so the proof of the next result is also omitted. In the statement of the theorem we will use the standard $q$-analog of $n$ which is $[n]=1+q+\cdots+q^{n-1}$.

Theorem 1.2 We have the following specific Wiener polynomials.

1. $W\left(K_{n} ; q\right)=\left(\begin{array}{c}n \\ 2\end{array}\right) q$.

2. $W\left(K_{m, n} ; q\right)=m n q+\left[\left(\begin{array}{c}m \\ 2\end{array}\right)+\left(\begin{array}{c}n \\ 2\end{array}\right)\right] q^{2}$.

3. $W\left(W_{n} ; q\right)=(2 n-2) q+\frac{(n-1)(n-4)}{2} q^{2}$.

4. $W(P ; q)=15 q+30 q^{2}$.

5. $W\left(P_{n} ; q\right)=(n-1) q+(n-2) q^{2}+\cdots+q^{n-1}=\frac{q}{1-q}(n-[n])$.

6. $W\left(C_{2 n} ; q\right)=(2 n)\left(q+q^{2}+\cdots+q^{n-1}\right)+n q^{n}=2 n([n]-1)+n q^{n}$.

7. $W\left(C_{2 n+1} ; q\right)=(2 n+1)\left(q+q^{2}+\cdots+q^{n}\right)=(2 n+1)([n]-1)$.

8. $W\left(Q_{n} ; q\right)=2^{n-1}\left[(1+q)^{n}-1\right]$.

Combining the previous theorem with number 5 of Theorem 1.1, we obtain the well-known Wiener indices of these graphs.

Theorem 1.3 We have the following specific Wiener indices.

1. $W\left(K_{n}\right)=\left(\begin{array}{l}n \\ 2\end{array}\right)$.

2. $W\left(K_{m, n}\right)=(m+n)^{2}-m n-m-n$.

3. $W\left(W_{n}\right)=(n-1)(n-2)$.

4. $W(P)=75$.

5. $W\left(P_{n}\right)=\left(\begin{array}{c}n+1 \\ 3\end{array}\right)$. 
6. $W\left(C_{2 n}\right)=(2 n)^{3} / 8$.

7. $W\left(C_{2 n+1}\right)=(2 n+2)(2 n+1)(2 n) / 8$.

8. $W\left(Q_{n}\right)=n 2^{2 n-2}$.

It would be interesting to see what various graph operations [7] do to the Wiener polynomial. Given graphs $G_{1}=\left(V_{1}, E_{1}\right)$ and $G_{2}=\left(V_{2}, E_{2}\right)$ with $\left|V_{i}\right|=n_{i}$ and $\left|E_{i}\right|=k_{i}$ for $i=1,2$ we define six new graphs formed from $G_{1}, G_{2}$.

1. Join: The graph $G_{1}+G_{2}$ has $V\left(G_{1}+G_{2}\right)=V_{1} \cup V_{2}$ and

$$
E\left(G_{1} \cup G_{2}\right)=E_{1} \cup E_{2} \cup\left\{u v: u \in V_{i}, v \in V_{2}\right\} .
$$

In the other five cases the vertex set is always $V_{1} \times V_{2}$.

2. Cartesian product: The graph $G_{1} \times G_{2}$ has edge set

$$
\left\{\left(u_{1}, u_{2}\right)\left(v_{1}, v_{2}\right): u_{1} v_{1} \in E_{1} \text { and } u_{2}=v_{2} \text { or } u_{2} v_{2} \in E_{2} \text { and } u_{1}=v_{1}\right\},
$$

3. Composition: The graph $G_{1}\left[G_{2}\right]$ has edge set

$$
\left\{\left(u_{1}, u_{2}\right)\left(v_{1}, v_{2}\right): u_{1} v_{1} \in E_{1} \text { or } u_{2} v_{2} \in E_{2} \text { and } u_{1}=v_{1}\right\},
$$

4. Disjunction: The graph $G_{1} \vee G_{2}$ has edge set

$$
\left\{\left(u_{1}, u_{2}\right)\left(v_{1}, v_{2}\right): u_{1} v_{1} \in E_{1} \text { or } u_{2} v_{2} \in E_{2} \text { or both }\right\},
$$

5. Symmetric difference: The graph $G_{1} \oplus G_{2}$ has edge set

$$
\left\{\left(u_{1}, u_{2}\right)\left(v_{1}, v_{2}\right): u_{1} v_{1} \in E_{1} \text { or } u_{2} v_{2} \in E_{2} \text { but not both }\right\},
$$

6. Tensor product: The graph $G_{1} \times G_{2}$ has edge set

$$
\left\{\left(u_{1}, u_{2}\right)\left(v_{1}, v_{2}\right): u_{1} v_{1} \in E_{1} \text { and } u_{2} v_{2} \in E_{2}\right\} .
$$

Taking a suggestion of Andreas Blass, it is sometimes more natural to express our results in terms of the ordered Wiener polynomial defined by

$$
\bar{W}(G ; q)=\sum_{(u, v)} q^{d(u, v)}
$$

where the sum is now over all ordered pairs $(u, v)$ of vertices, including those where $u=v$. Thus

$$
\bar{W}(G ; q)=2 W(G ; q)+|V(G)| .
$$

Also it will be convenient to have a variable for the non-edges in $G_{i}$ so let $\bar{k}_{i}=$ $\left(\begin{array}{c}n_{i} \\ 2\end{array}\right)-k_{i}$ for $i=1,2$. 
Proposition 1.4 Suppose $G_{1}$ and $G_{2}$ are connected and nontrivial (not equal to $\left.K_{1}\right)$. Then with the preceding notation

1. $W\left(G_{1}+G_{2} ; q\right)=\left(k_{1}+k_{2}+n_{1} n_{2}\right) q+\left(\bar{k}_{1}+\bar{k}_{2}\right) q^{2}$,

2. $\bar{W}\left(G_{1} \times G_{2} ; q\right)=\bar{W}\left(G_{1} ; q\right) \bar{W}\left(G_{2} ; q\right)$,

3. $W\left(G_{1}\left[G_{2}\right] ; q\right)=n_{1}\left(k_{2} q+\bar{k}_{2} q^{2}\right)+n_{2}^{2} W\left(G_{1} ; q\right)$,

4. $W\left(G_{1} \vee G_{2} ; q\right)=\left(n_{1}^{2} k_{2}+n_{2}^{2} k_{1}-2 k_{1} k_{2}\right) q+\left(n_{1} \bar{k}_{2}+n_{2} \bar{k}_{1}+2 \bar{k}_{1} \bar{k}_{2}\right) q^{2}$,

5. $W\left(G_{1} \oplus G_{2} ; q\right)=\left(n_{1} k_{2}+n_{2} k_{1}+2 k_{1} \bar{k}_{2}+2 k_{2} \bar{k}_{1}\right) q+\left(n_{1} \bar{k}_{2}+n_{2} \bar{k}_{1}+2 k_{1} k_{2}+\right.$ $\left.2 \bar{k}_{1} \bar{k}_{2}\right) q^{2}$.

Proof. In each part of this proof let $d_{1}, d_{2}$ and $d$ denote the distance functions in $G_{1}, G_{2}$ and the graph formed from $G_{1}$ and $G_{2}$, respectively.

1. In $G_{1}+G_{2}$ all pairs of vertices are either at distance one or two. If $d(u, v)=1$ then either $u v \in E_{1}$ or $u v \in E_{2}$ or $u \in E_{1}, v \in E_{2}$. This gives the linear coefficient in $W\left(G_{1}+G_{2} ; q\right)$. The other term is gotten by counting the remaining vertex pairs.

2. A geodesic for an ordered pair $\left(\left(u_{1}, u_{2}\right),\left(v_{1}, v_{2}\right)\right)$ is obtained by following a geodesic in $G_{1}$ for $\left(u_{1} u_{2}, v_{1} u_{2}\right)$ and then one in $G_{2}$ for $\left(v_{1} u_{2}, v_{1} v_{2}\right)$. The stated formula for $\bar{W}\left(G_{1} \times G_{2} ; q\right)$ follows.

3. First consider pairs $\left\{\left(u_{1}, u_{2}\right),\left(v_{1}, v_{2}\right)\right\}$ with $u_{1}=v_{1}$. Then

$$
d\left(\left(u_{1}, u_{2}\right),\left(u_{1}, v_{2}\right)\right)= \begin{cases}1 & \text { if } u_{2} v_{2} \in E_{2} \\ 2 & \text { if } u_{2} v_{2} \notin E_{2}\end{cases}
$$

with a geodesic in the second case being $\left(u_{1}, u_{2}\right),\left(w_{1}, v_{2}\right),\left(u_{1}, v_{2}\right)$ where $w_{1}$ is any vertex adjacent to $u_{1}$ in $G_{1}$. These vertex pairs contribute the first two terms in the sum for $W\left(G_{1}\left[G_{2}\right] ; q\right)$. If $u_{1} \neq v_{1}$ then $d\left(\left(u_{1}, u_{2}\right),\left(v_{1}, v_{2}\right)\right)=d_{1}\left(u_{1}, v_{1}\right)$ since a $u_{1}$ to $v_{1}$ geodesic in $G_{1}$ gives rise to one in $G_{1}\left[G_{2}\right]$ by adding a second component equal to $u_{2}$ for the first vertex and to $v_{2}$ for all other vertices of the geodesic.

4. This is similar to the previous proof where

$$
d\left(\left(u_{1}, u_{2}\right),\left(v_{1}, v_{2}\right)\right)= \begin{cases}1 & \text { if } u_{1} v_{1} \in E_{1} \text { or } u_{2} v_{2} \in E_{2} \\ 2 & \text { else }\end{cases}
$$

with a geodesic in the second case being $\left(u_{1}, u_{2}\right),\left(w_{1}, w_{2}\right),\left(u_{1}, v_{2}\right)$ where $u_{1} w_{1} \in E_{1}$ and $w_{2} v_{2} \in E_{2}$.

5. This is again similar to the previous two proofs with

$$
d\left(\left(u_{1}, u_{2}\right),\left(v_{1}, v_{2}\right)\right)= \begin{cases}1 & \text { if exactly one of } u_{1} v_{1} \in E_{1} \text { or } u_{2} v_{2} \in E_{2} \\ 2 & \text { else. }\end{cases}
$$


In the second case, how to choose the middle vertex of the geodesic depends on whether the neighborhoods of $u_{i}$ and $v_{i}$ are the same or not, $i=1,2$, as well as on whether both $u_{1} v_{1} \in E_{1}$ and $u_{2} v_{2} \in E_{2}$ or neither.

As a corollary, we can rederive some of the $W(G ; q)$ from Theorem 1.2 as well as the ordered Wiener polynomial of the grid $P_{m} \times P_{n}$.

Corollary 1.5 We have the following specific Wiener polynomials

1. $W\left(K_{m, n} ; q\right)=m n q+\left[\left(\begin{array}{c}m \\ 2\end{array}\right)+\left(\begin{array}{c}n \\ 2\end{array}\right)\right] q^{2}$.

2. $W\left(W_{n} ; q\right)=(2 n-2) q+\frac{(n-1)(n-4)}{2} q^{2}$.

3. $\bar{W}\left(Q_{n} ; q\right)=2^{n}(1+q)^{n}$.

4. $\bar{W}\left(P_{m} \times P_{n}\right)=\frac{1}{(1-q)^{2}}((1+q) m-2 q[m])((1+q) n-2 q[n])$.

Proof. For the first two polynomials use part 1 of Theorem 1.4 as well as the fact that $W_{n}=C_{n-1}+K_{1}$ and $K_{m, n}=\bar{K}_{m}+\bar{K}_{n}$ where $\bar{K}_{i}$ is the completely disconnected graph on $i$ vertices. For the last two polynomials use part 2 of the same theorem along with the $n$-fold product $Q_{n}=K_{2} \times \cdots \times K_{2}$. Note that in addition one needs $\bar{W}\left(K_{2} ; q\right)=2+2 q$ and $\bar{W}\left(P_{n} ; q\right)=2 W\left(P_{n} ; q\right)+n=$ $\frac{1}{1-q}((1+q) n-2 q[n])$.

Now we will find the Wiener polynomial of a dendrimer, which will take considerably more work.

\section{The Wiener polynomial of a dendrimer}

The $d$-ary dendrimer on $n$ nodes, $D_{n, d}$, is defined inductively as follows. The tree $D_{1, d}$ consists of a single node labeled 1 . The tree $D_{n, d}$ has vertex set $\{1,2, \ldots, n\}$. It is obtained by attaching a leaf $n$ to the smallest numbered node of $D_{n-1, d}$ which has degree $\leq d$. It is convenient to consider $D_{n, d}$ as if it were rooted at vertex number 1 with the nodes at each level ordered left to right in increasing order of their numbering. Thus in a typical tree the root has $d+1$ children while every other internal vertex (possibly with one exception) has $d$. The dendrimer $D_{17,2}$ is pictured in Figure 1

Define $n_{k}$ (respectively, $m_{k}$ ) to be the number of vertices in the $d$-ary dendrimer with exactly one descendant of vertex 2 (respectively, of vertex 3 ) at level $k+1$. Thus

$$
n_{k}=2+(d+1) \frac{d^{k}-1}{d-1} \text { and } m_{k}=3+(2 d) \frac{d^{k}-1}{d-1} .
$$




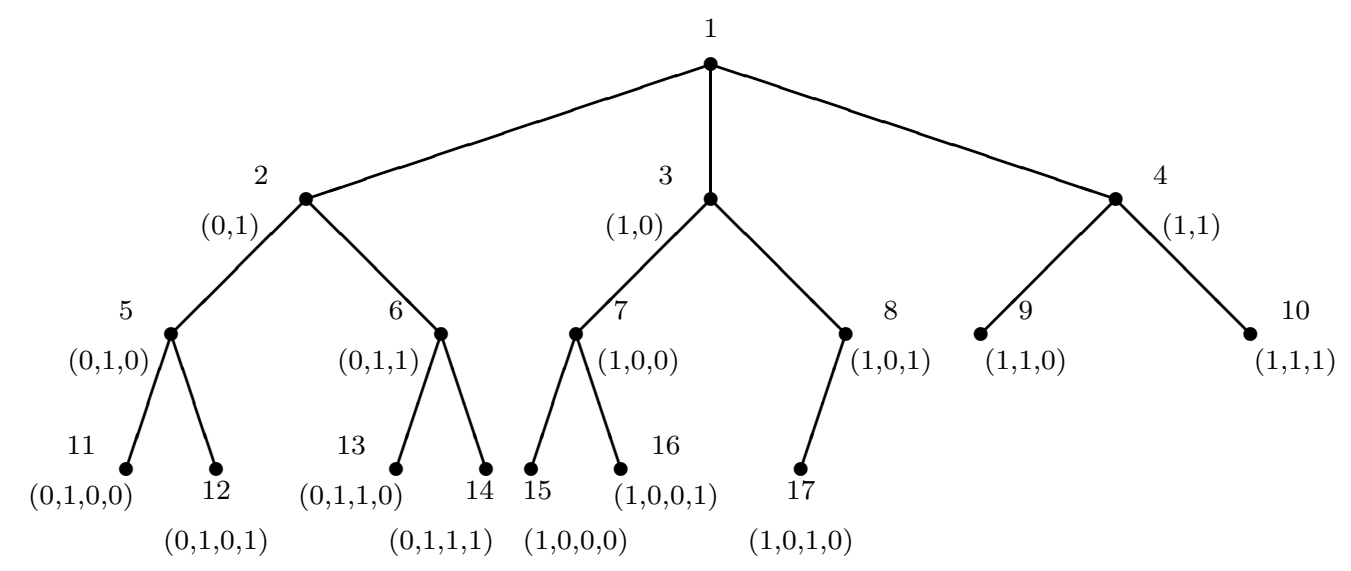

Figure 1: The dendrimer $D_{17,2}$

The tree in Figure 1 has $n_{0}=2, n_{1}=5, n_{2}=11$ and $m_{0}=3, m_{1}=7, m_{2}=15$.

To describe $W\left(D_{n, d} ; q\right)$ we will also need to give each vertex $m>1$ a label $\lambda(m)$ in addition to its number. Specifically, if $n_{k} \leq m<n_{k+1}$ then

$$
\lambda(m)=\left(l_{k+1}, l_{k}, \cdots, l_{0}\right) \text { where } \sum_{i=0}^{k+1} l_{i} d^{i}=n-n_{k}+(d-1) d^{k}, 0 \leq l_{i}<d \forall i
$$

so that the $l_{i}$ are the digits in the base $d$ expansion of $n-n_{k}+(d-1) d^{k}$ (possibly with a leading zero). Thus all the vertices at level $k+1$ have labels which are consecutive base $d$ from $(0, d-1, \overbrace{0, \ldots, 0}^{k})$ to $(1, \overbrace{d-1, \ldots, d-1}^{k+1})$. This implies that if $m$ has label $\lambda(m)=\left(l_{k+1}, l_{k}, \cdots, l_{0}\right)$ then $m$ 's children are labeled left to right with $\left(l_{k+1}, l_{k}, \cdots, l_{0}, 0\right)$ to $\left(l_{k+1}, l_{k}, \cdots, l_{0}, d-1\right)$. To illustrate, the labels of the vertices of $D_{17,2}$ are also given in Figure 1.

To find $W\left(D_{n, d} ; q\right)$, we first consider the corresponding difference polynomials,

$$
\Delta W\left(D_{n, d} ; q\right)=W\left(D_{n, d} ; q\right)-W\left(D_{n-1, d} ; q\right) .
$$

So if $\Delta\left(W\left(D_{n, d} ; q\right)\right)=\sum_{i} c_{i} q^{i}$ then $c_{i}$ is the number of vertices in $D_{n, d}$ that are at distance $i$ from $n$.

Lemma 2.1 Suppose $\lambda(n)=\left(l_{k+1}, l_{k}, \cdots, l_{0}\right)$. Then

$$
\Delta W\left(D_{n, d} ; q\right)=\sum_{i=0}^{k} d^{i} q^{2 i+1}+\sum_{i=0}^{k^{\prime}} d^{i}\left(l_{i}+1\right) q^{2 i+2}
$$


where $k^{\prime}=k-1$ or $k$ for $n_{k} \leq n<m_{k}$ or $m_{k} \leq n<n_{k+1}$, respectively.

Proof. We will do the case $n_{k} \leq n<m_{k}$, the other being similar. Suppose first that $n=n_{k}$ so that $l_{0}=\ldots=l_{k-1}=0$. Thus we wish to show

$$
\Delta W\left(D_{n_{k}, d} ; q\right)=q+q^{2}+d q^{3}+d q^{4}+\cdots+d^{k} q^{2 k+1} .
$$

Every vertex at distance $i-1$ from $n_{k-1}$ in $D_{n_{k-1}, d}$ is at distance $i$ from $n_{k}$ in $D_{n_{k}, d}$. When $i=2 j$ is even, this accounts for all the vertices at distance $2 j$ from $n_{k}$ and so

$$
\left[q^{2 j}\right] \Delta W\left(D_{n_{k}, d} ; q\right)=\left[q^{2 j-1}\right] \Delta W\left(D_{n_{k-1}, d} ; q\right)=d^{j-1}
$$

by induction. When $i=2 j+1>1$ then any leaves of $D_{n_{k}, d}$ which are descendants of $n_{k-j-1}$ but not of $n_{k-j}$ are also at distance $2 j+1$ from $n_{k}$. (If $j=k$ then let $n_{-1}=1$.) So by induction

$$
\begin{aligned}
{\left[q^{2 j+1}\right] \Delta W\left(D_{n_{k}, d} ; q\right) } & =\left[q^{2 j}\right] \Delta W\left(D_{n_{k-1}, d} ; q\right)+\#(\text { leaves at distance } 2 i+1) \\
& = \begin{cases}d^{j-1}+d^{j-1} & \text { if } j<k \\
0+d^{k} & \text { if } j=k\end{cases} \\
& =d^{j} \text { for all } j .
\end{aligned}
$$

This completes the proof when $n=n_{k}$.

Now suppose that $n_{k}<n<m_{k}$. We will construct a function $f$ that sets up a bijection between vertices at distance $i$ from vertex $n$ in $D_{n, d}$ and those at distance $i$ from vertex $n^{\prime}$ in $D_{n^{\prime}, d}$ where $n_{k} \leq n^{\prime}<n$ and then use induction. The bijection will hold for all $i, 0 \leq i \leq 2 k+1$, except for $i=2 j$ where $j$ will be determined shortly.

All vertices satisfying $n_{k}<n<m_{k}$ are descendents of vertex 2 . However, since $n \neq n_{k}$ the unique 2 to $n$ path contains an edge $u v$ where $v$ is not the leftmost child of $u$. Let $u$ be the lowest such vertex and define

$$
\begin{aligned}
j & =\text { distance from } u \text { to } n, \\
v^{\prime} & =\text { child of } u \text { just to the left of } v, \\
n^{\prime} & =\text { leftmost lowest descendant of } v^{\prime} .
\end{aligned}
$$

For a schematic sketch of this situation for $d=2$, see Figure 2 .

It follows from the definitions and the way in which children of a vertex are labeled that

$$
\lambda(n)=\left(l_{k+1}, \ldots, l_{0}\right)=\left(l_{k+1}, \ldots, l_{j}, l, 0, \ldots, 0\right)
$$

and

$$
\lambda\left(n^{\prime}\right)=\left(l_{k+1}^{\prime}, \ldots, l_{0}^{\prime}\right)=\left(l_{k+1}, \ldots, l_{j}, l-1,0, \ldots, 0\right)
$$




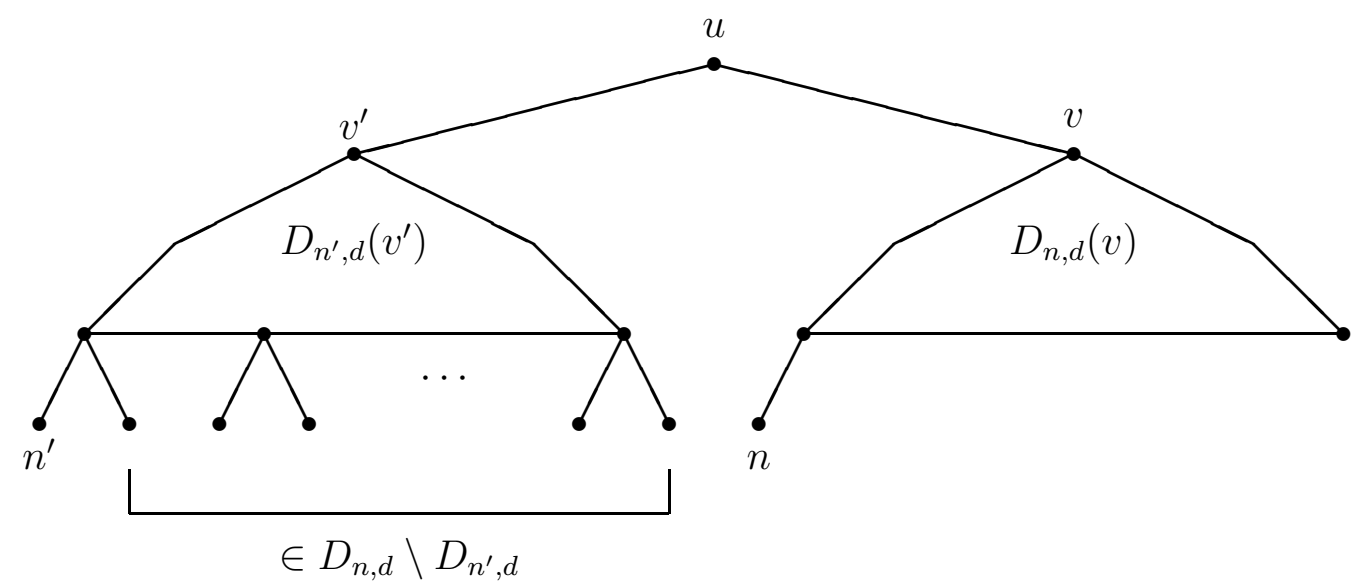

Figure 2: Location of $u, v, v^{\prime}, n$ and $n^{\prime}$ when $d=2$

where $l>0$. So $l_{i}=l_{i}^{\prime}$ if $i \neq j-1$ and $l_{j-1}$ only enters into the coefficient of $q^{2 j}$. Construction of the bijection $f$ will establish that $\left[q^{i}\right] \Delta W\left(D_{n, d} ; q\right)=$ $\left[q^{i}\right] \Delta W\left(D_{n^{\prime}, d} ; q\right)$ for $i \neq 2 j$. Thus induction combined with the equations for $\lambda(n)$ and $\lambda\left(n^{\prime}\right)$ will complete the proof in this case.

Construct $f$ as follows. Given any ordered tree $T$ and one of its vertices $v$, then we let $T(v)$ denote the subtree of $T$ consisting of $v$ and all its descendants. Note that there is a unique isomorphism of ordered trees $g: D_{n, d}(v) \rightarrow D_{n^{\prime}, d}\left(v^{\prime}\right)$. Also note that all vertices of $D_{n, d} \backslash D_{n^{\prime}, d}$ (other than $n$ itself) are leaves at distance $2 j$ from $n$. So if $w$ is any vertex of $D_{n^{\prime}, d} \cup n$ then let

$$
f(w)= \begin{cases}g(w) & \text { if } w \in D_{n, d}(v) \\ g^{-1}(w) & \text { if } w \in D_{n^{\prime}, d}\left(v^{\prime}\right) \\ w & \text { else. }\end{cases}
$$

Thus $d(n, w)=d\left(n^{\prime}, f(w)\right)$ : For $w \in D_{n, d}(v) \cup D_{n^{\prime}, d}\left(v^{\prime}\right)$ this follows because $g$ is an isomorphism. For any other $w$, the unique $n$ to $w$ and $n^{\prime}$ to $w$ paths both go through $u$, so $d(n, w)=d\left(n^{\prime}, w\right)$. The function $f$ is also clearly bijective, so we are done when $i \neq 2 j$.

To complete the proof, note that $f$ restricts to an injection from the vertices at distance $2 j$ from $n^{\prime}$ in $D_{n^{\prime}, d}$ into those at distance $2 j$ from $n$ in $D_{n, d}$. The only remaining $w$ with $d(w, n)=2 j$ are the leaves of $D_{n, d}\left(v^{\prime}\right)$ which are $d^{j-1}$ in number. So by induction and equations (2) and (3)

$$
\left[q^{2 j}\right] \Delta W\left(D_{n, d} ; q\right)=\left[q^{2 j}\right] \Delta W\left(D_{n^{\prime}, d} ; q\right)+d^{j-1}=d^{j-1}(l+1)
$$


as desired.

We are now in a position to compute $W\left(D_{n, d} ; q\right)$. In the following theorem $\lfloor\cdot\rfloor$ denotes the floor (round down) function.

Theorem 2.2 Suppose $\lambda(n)=\left(l_{k+1}, l_{k}, \cdots, l_{0}\right)$ and define

$$
\lambda_{i}(n)=l_{0}+l_{1} d+\cdots l_{i-1} d^{i-1} .
$$

If $n_{k} \leq n<m_{k}$ then

$$
\begin{aligned}
W\left(D_{n, d} ; q\right) & =\sum_{i=0}^{k} d^{i}\left(n-n_{i}+1\right) q^{2 i+1}+ \\
& \sum_{i=0}^{k^{\prime}}\left(d^{2 i}\left\lfloor\frac{n-m_{i}}{d^{i+1}}\right\rfloor\left(\begin{array}{c}
d+1 \\
2
\end{array}\right)+d^{2 i}\left(\begin{array}{c}
l_{i}+1 \\
2
\end{array}\right)+d^{i}\left(l_{i}+1\right)\left(\lambda_{i}(n)+1\right)\right) q^{2 i+2} .
\end{aligned}
$$

where $k^{\prime}=k-1$ or $k$ for $n_{k} \leq n<m_{k}$ or $m_{k} \leq n<n_{k+1}$, respectively.

Proof. Since $W\left(D_{1, d} ; q\right)=0$, we have $W\left(D_{n, d} ; q\right)=\sum_{m=2}^{n} \Delta W\left(D_{m, d} ; q\right)$. Thus the theorem will follow from Lemma 2.1 and summation. Summing the coefficients of $q^{2 i+1}$ in the various $\Delta W\left(D_{m, d} ; q\right)$ is easy since the nonzero ones are all equal to $d^{i}$. The nonzero $\left[q^{2 i+2}\right] \Delta W\left(D_{m, d} ; q\right)$ are periodic with period $d^{i+1}$. In fact this periodic sequence is

$$
d^{i}, \cdots, d^{i}, 2 d^{i}, \cdots, 2 d^{i}, \cdots, d^{i+1}, \cdots, d^{i+1}
$$

where each block of equal values is of size $d^{i}$. Thus the sum of a complete period is $d^{2 i}\left(\begin{array}{c}d+1 \\ 2\end{array}\right)$ and the term containing this expression in $\left[q^{2 i+2}\right] W\left(D_{n, d} ; q\right)$ comes from summing all complete periods prior to the (perhaps partial) period containing $c=\left[q^{2 i+2}\right] \Delta W\left(D_{n, d} ; q\right)$. The term $d^{2 i}\left(\begin{array}{c}l_{i}+1 \\ 2\end{array}\right)$ comes from summing all complete blocks of the last period prior to the (perhaps partial) block containing $c$. Finally, $d^{i}\left(l_{i}+1\right)\left(\lambda_{i}(n)+1\right)$ is the contribution of the block containing $c$.

We will now rederive the Wiener index of a complete dendrimer, as was first done in [6]. A complete dendrimer is $D_{n, d}$ where $n=n_{k}-1$, i.e., as an ordered tree it is complete to level $k$. The reader should be warned that in [6] they only consider complete dendrimers and index them with two parameters that are different from ours. We have

$$
\lambda\left(n_{k}-1\right)=(1, \overbrace{d-1, \ldots, d-1}^{k})
$$

and

$$
\lambda_{i}\left(n_{k}-1\right)=d^{i}-1
$$


for $i<k$. Substituting these values into the formulas of Theorem 2.2 gives

$$
\begin{aligned}
W\left(D_{n_{k}-1, d} ; q\right)= & \sum_{i=0}^{k-1} d^{i} \cdot d^{i}(d+1) \frac{d^{k-i}-1}{d-1} q^{2 i+1} \\
& +\sum_{i=0}^{k-1}\left[d^{2 i}(d+1) \frac{d^{k-i-1}-1}{d-1}\left(\begin{array}{c}
d+1 \\
2
\end{array}\right)+d^{2 i}\left(\begin{array}{l}
d \\
2
\end{array}\right)+d^{i} \cdot d \cdot d^{i}\right] q^{2 i+2} \\
= & \sum_{i=0}^{k-1} d^{2 i}(d+1) \frac{d^{k-i}-1}{d-1} q^{2 i+1} \\
& +\sum_{i=0}^{k-1} d^{2 i}\left(\begin{array}{c}
d+1 \\
2
\end{array}\right)\left[(d+1) \frac{d^{k-i-1}-1}{d-1}+1\right] q^{2 i+2} .
\end{aligned}
$$

Taking the derivative of the previous equation and setting $t=1$ gives the Wiener index according to Theorem 1.1, number 5. To evaluate the summations, use

$$
\sum_{i=0}^{k-1}(i+1) d^{i}=\frac{k d^{k+1}-(k+1) d^{k}+1}{(d-1)^{2}}
$$

and its variants repeatedly to obtain

$$
\begin{aligned}
W\left(D_{n_{k}-1, d}\right)= & \frac{d+1}{d-1}\left[\frac{(2 k-1) d^{2 k+1}-(2 k+1) d^{2 k}+d^{k+1}+d^{k}}{(d-1)^{2}}\right. \\
& \left.-\frac{(2 k-1) d^{2 k+2}-(2 k+1) d^{2 k}+d^{2}+1}{\left(d^{2}-1\right)^{2}}\right] \\
& +\frac{d(d+1)^{2}}{d-1}\left[\frac{k d^{2 k}-(k+1) d^{2 k-1}+d^{k-1}}{(d-1)^{2}}\right. \\
& \left.-\frac{k d^{2 k+2}-(k+1) d^{2 k}+1}{\left(d^{2}-1\right)^{2}}\right]+d(d+1) \frac{k d^{2 k+2}-(k+1) d^{2 k}+1}{\left(d^{2}-1\right)^{2}} .
\end{aligned}
$$

Simplification of the above expression yields the following result which is equivalent to equation (9) of [6] after a change of variables.

Corollary 2.3 The Wiener index of a complete dendrimer is

$$
W\left(D_{n_{k}-1, d}\right)=\frac{d^{2 k}\left[k d^{3}+(k-2) d^{2}-(k+3) d-(k+1)\right]+2 d^{k}(d+1)^{2}-(d+1)}{(d-1)^{3}} .
$$

\section{Unimodality}

We say a sequence $\left(a_{m}\right)_{m \geq 0}$ is unimodal if, for some index $k$

$$
a_{0} \leq a_{1} \leq \ldots \leq a_{k} \geq a_{k+1} \geq a_{k+2} \geq \ldots
$$


Unimodal sequences appear in many areas of mathematics. For a survey, see Stanley's article [11]. We will show that the coefficients of $W\left(D_{n, d}\right)$ are unimodal. First, however, we will need a general result about sequences and their differences. The difference sequence of $\left(a_{m}\right)_{m \geq 0}$ is $\left(\Delta a_{m}\right)_{m \geq 1}$ where $\Delta a_{m}=a_{m}-a_{m-1}$.

Proposition 3.1 Suppose $\left(a_{m}\right)_{m \geq 0}$ and $\left(b_{m}\right)_{m \geq 0}$ are two sequences with $\Delta a_{m} \leq$ $\Delta b_{m}$ for all $m$ in some interval $I$ of integers. If $a_{M} \leq b_{M}$ for some $M \in I$ then $a_{m} \leq b_{m}$ for all $m \geq M$ with $m \in I$. On the other hand, if $a_{M} \geq b_{M}$ for some $M \in I$ then $a_{m} \geq b_{m}$ for all $m \leq M$ with $m \in I$.

Proof. We will consider the case $a_{M} \leq b_{M}$, the other being similar. Using the given inequalities we have, for $m \geq M$,

$$
a_{m}=a_{M}+\sum_{k=M+1}^{m} \Delta a_{k} \leq b_{M}+\sum_{k=M+1}^{m} \Delta b_{k}=b_{m}
$$

as desired.

We will need coefficients of $W\left(D_{n, d} ; q\right)$ to play the roles of $a_{M}$ and $b_{M}$ in the previous Proposition.

Lemma 3.2 Let $p_{k}=m_{k}+2 d^{k}-1$ and set $W(t)=W\left(D_{p_{k}, d} ; q\right)$. Then

$$
\left[q^{2 k}\right] W(t) \leq\left[q^{2 k+1}\right] W(t)=\left[q^{2 k+2}\right] W(t) .
$$

Proof. Note that by the choice of $p_{k}$ we have

$$
\lambda\left(p_{k}\right)=(1,1, \overbrace{d-1, \ldots, d-1}^{k})
$$

and so

$$
\lambda_{i}\left(p_{k}\right)=d^{i}-1
$$

for $i \leq k$. Using Theorem 2.2 and the previous two equations we get

$$
\left[q^{2 k}\right] W(t)=d^{2 k-2}\left\lfloor\frac{p_{k}-m_{k-1}}{d^{k}}\right\rfloor\left(\begin{array}{c}
d+1 \\
2
\end{array}\right)+d^{2 k-2}\left(\begin{array}{l}
d \\
2
\end{array}\right)+d^{k-1} \cdot d \cdot d^{k-1} .
$$

But $p_{k}-m_{k-1}=m_{k}-m_{k-1}+2 d^{k}-1=4 d^{k}-1$ so

$$
\left[q^{2 k}\right] W(t)=d^{2 k-1}\left(3 \frac{d+1}{2}+\frac{d-1}{2}+1\right)=2 d^{2 k-1}(d+1)
$$

Using Theorem 2.2 again we obtain

$$
\left[q^{2 k+1}\right] W(t)=d^{k}\left(p_{k}-n_{k}+1\right)=d^{k}\left(m_{k}-n_{k}+2 d^{k}\right)=3 d^{2 k} .
$$


Comparison of equations (6) and (7) and the fact that $d \geq 2$ yield the inequality in the statement of lemma.

One last application of Theorem 2.2 together with equations (4) and (5) gives $\left[q^{2 k+2}\right] W(t)=d^{2 k}\left\lfloor\frac{p_{k}-m_{k}}{d^{k+1}}\right\rfloor\left(\begin{array}{c}d+1 \\ 2\end{array}\right)+d^{2 k}\left(\begin{array}{l}2 \\ 2\end{array}\right)+d^{k} \cdot 2 \cdot d^{k}=0+d^{2 k}+2 d^{2 k}=3 d^{2 k}$.

But this is the same as the value obtained in equation (7), so we are done with the proof.

We now put the various pieces together to get the promised theorem.

Theorem 3.3 Let $W(t)=W\left(D_{n, d} ; q\right)$. Then the coefficients of $W(t)$ are unimodal. Furthermore, for $n_{k} \leq n<p_{k}$ either $\left[q^{2 k}\right] W(t)$ or $\left[q^{2 k+1}\right] W(t)$ is a maximum. On the other hand, for $p_{k} \leq n<n_{k+1}$ we have a maximum at $\left[q^{2 k+2}\right] W(t)$ so that in this case the sequence is increasing.

Proof. We will only consider the case $n_{k} \leq n<p_{k}$. The reader can easily fill in the details in the other one. Since $\operatorname{deg} W(t) \leq 2 k+2$ it suffices to show that the following two equations hold:

$$
\begin{aligned}
{\left[q^{i}\right] W(t) } & \leq\left[q^{i+1}\right] W(t) \text { for } i<2 k, \text { and } \\
{\left[q^{2 k+1}\right] W(t) } & \geq\left[q^{2 k+2}\right] W(t)
\end{aligned}
$$

For the first inequality, fix $i$ and consider the sequence whose terms are given by $a_{m}=\left[q^{i}\right] W\left(D_{m, d} ; q\right)$. So in particular $a_{n}=\left[q^{i}\right] W(t)$. Similarly define $b_{m}=$ $\left[q^{i+1}\right] W\left(D_{m, d} ; q\right)$ so that $b_{n}=\left[q^{i+1}\right] W(t)$. Lemma 2.1 shows that these two sequences satisfy the supposition of Proposition 3.1 for $m \geq m_{\lfloor i / 2\rfloor}$. By the lemma

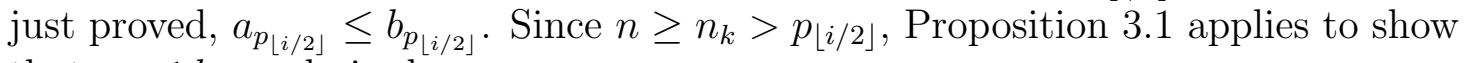
that $a_{n} \leq b_{n}$ as desired.

The second inequality is clearly true for $n_{k} \leq n<m_{k}$ since then $\left[q^{2 k+2}\right] W(t)=$ 0 . If $m_{k} \leq n<p_{k}$, then consider the sequences defined by $a_{m}=\left[q^{2 k+1}\right] W\left(D_{m, d} ; q\right)$ and $b_{m}=\left[q^{2 k+2}\right] W\left(D_{m, d} ; q\right)$. These satisfy the hypothesis of Proposition 3.1 for $m \geq m_{k}$. By the previous lemma $a_{p_{k}}=b_{p_{k}}$. So, since $n<p_{k}$, we can apply Proposition 3.1 to get $a_{n} \geq b_{n}$ which concludes the proof.

\section{Comments and open questions}

Since the Wiener polynomial is a new graphical invariant, there are many questions one could ask about it. We summarize some of them in this section 
(I) The reader has probably noticed that we did not provide a formula for $W\left(G_{1} \times G_{2} ; q\right)$ in terms of $W\left(G_{1} ; q\right)$ and $W\left(G_{2} ; q\right)$. It would be interesting to fill this gap in the list of Wiener polynomials related to graph operations.

(II) A referee pointed out that the generating function for the Wiener polynomial of the complete dendrimer has a nice form. It can be obtained algebraically from the equation for $W\left(D_{n_{k}-1, d} ; q\right)$ given at the end of section 2 , but the referee asked for a combinatorial proof. We give such a demonstration next.

Proposition 4.1 The generating function for $W\left(D_{n_{k}-1, d} ; q\right)$ is

$$
\sum_{k \geq 1} W\left(D_{n_{k}-1, d} ; q\right) z^{k}=z \frac{(d+1) q+\left(\begin{array}{c}
d+1 \\
2
\end{array}\right) q^{2}(1+z)}{(1-z)(1-d z)\left(1-d^{2} q^{2} z\right)} .
$$

Proof. Let $T_{k}=D_{n_{k}-1, d}$ and $F(z)=\sum_{k \geq 1} W\left(T_{k} ; q\right) z^{k}$. Consider $T_{k-1}$ as embedded in $T_{k}$ so that their roots coincide. Then $(1-z) F(z)$ is the generating function for all $u$ to $v$ paths in $T_{k}$ such that at least one of $u, v$ is a leaf, $u \neq v$. Now there is a $d^{2}$-to-1 mapping from paths $P$ of length $l$ in $T_{k}$ to paths $P^{\prime}$ of length $l-2$ in $T_{k-1}$ gotten by removing the two endpoints of $P$. Furthermore one of the endpoints of $P$ is a leaf iff one of the endpoints of $P^{\prime}$ is a leaf. So $\left(1-d^{2} q^{2} z\right)(1-z) F(z)$ is the generating function for all paths in $T_{k}$ of length one or two with at least one endpoint a leaf. Now if $k>2$ then $T_{k}$ has exactly $d$ times as many such paths as $T_{k-1}$. So $(1-d z)\left(1-d^{2} q^{2} z\right)(1-z) F(z)$ is a polynomial of degree 2 which is easy to compute directly, giving the numerator of the fraction in the statement of the proposition.

(III) The Wiener polynomial refines the Wiener index since it gives information about how many pairs of vertices are at a given distance $i$, not just the sum of all distances. One can also refine the Wiener polynomial itself as follows. Define the Wiener polynomial of a graph $G$ relative to a vertex $v$ by

$$
W_{v}(G ; q)=\sum_{w \in V} q^{d(v, w)} .
$$

The next result is immediate from the definitions and will be useful latter.

Proposition 4.2 We have the following relationship between the ordered and relative Wiener polynomials

$$
\bar{W}(G ; q)=\sum_{v} W_{v}(G ; q) .
$$

Furthermore, if $G$ is vertex transitive then for any vertex $v \in G$ we have

$$
\bar{W}(G ; q)=|V| W_{v}(G ; q) .
$$


(IV) In certain cases, Wiener polynomial is closely related to a polynomial that appears in the theory of Coxeter groups. We will follow the text of Humphreys [9] in terms of definitions and notation. Let $(W, S)$ be a Coxeter system and let $T=\left\{w s w^{-1}: w \in W, s \in S\right\}$. There should be no confusion between the notation for a Coxeter group $W$ and the one for the Wiener invariants since the latter is always followed by a parenthesized expression. The absolute length of $w \in W$ is the minimum number $k$ such that

$$
w=t_{1} t_{2} \cdots t_{k} \quad \text { where } t_{i} \in T \forall i .
$$

We write $\hat{l}(w)=k$ in this case. The absolute length function differs from the ordinary length function in that the factors in the product for $w$ are required to be in $T$ rather than in $S$. Recently Barcelo, Garsia and Goupil [1] have found a beautiful connection between absolute length and NBC bases.

Our interest is in the Poincaré polynomial of $W$ which is defined by

$$
\Pi(W ; q)=\sum_{w \in W} q^{\hat{l}(w)} .
$$

It is related to the extended Wiener polynomial as follows. We will define a graph $G_{W}$ associated with any Coxeter group. The vertices of $G_{W}$ are the elements of $W$ and we connect $v$ and $w$ by an edge if $v=w t$ for some $t \in T$. This graph is related to the strong Bruhat ordering of $W$. Note that $\hat{l}(w)=d(1, w)$ where 1 is the identity element of $W$ and distance is taken in $G_{W}$. Combining this observation with Proposition 4.2 and the easily proved fact that $G_{W}$ is vertex transitive, we obtain the connection between the two polynomials.

Proposition 4.3 If $W$ is a finite Coxeter group and $G_{W}$ is the corresponding graph then

$$
\bar{W}\left(G_{W} ; q\right)=|W| \Pi(W ; q) .
$$

The next theorem is well known, see the book of Orlik and Terao [10].

Theorem 4.4 If $W$ is a finite Coxeter group then its Poincaré polynomial factors as

$$
\Pi(W ; q)=\prod_{e}(1+e q)
$$

where the product is over all exponents e of $W$. In particular, the roots of $\Pi(W ; q)$ are all negative rational numbers.

The comment about the roots of $\Pi(W ; q)$ relates to the concept of unimodality as follows. We say a sequence $\left(a_{m}\right)_{m \geq 0}$ is $\log$ concave if $a_{m}^{2} \geq a_{m-1} a_{m+1}$ for all $m \geq 1$. The relationship between these three concepts is easy to prove (see [11]). 
Proposition 4.5 Let $\left(a_{m}\right)_{0 \leq m \leq M}$ be a sequence of positive numbers and let $f(q)=$ $\sum_{m=0}^{M} a_{m} q^{m}$ be the corresponding polynomial.

1. If $f(q)$ factors over the negative rationals then $\left(a_{m}\right)$ is log concave.

2. If $\left(a_{m}\right)$ is $\log$ concave then it is also unimodal.

This brings up three questions.

1. For which graphs $G$ is the coefficient sequence of $W(G ; q)$ unimodal?

2. For which graphs $G$ is the coefficient sequence of $W(G ; q) \log$ concave?

3. For which graphs $G$ does $\bar{W}(G ; q)$ factor over the negative rationals.

Note that both the graphs $G_{W}$ and $Q_{n}$ satisfy the last condition which is the strongest of the three.

(V) There are two theorems that are useful for computing the Wiener index of a tree that we have been unable to find analogs for in the case of the Wiener polynomial. The first is due to Wiener himself [12].

Theorem 4.6 Let $T$ be a tree and let $e$ be an edge in $E=E(T)$. Let $n_{1}(e)$ and $n_{2}(e)$ be the number of vertices in the two components of $T-e$. Then

$$
W(T)=\sum_{e \in E} n_{1}(e) n_{2}(e)
$$

The second is a result of Gutman [4]. It is useful when the tree in question has few vertices of high degree.

Theorem 4.7 Let $T$ be a tree and let $v$ be a vertex in $V=V(T)$ with $\operatorname{deg} v \geq 3$. Let $n_{1}(v), \ldots, n_{\operatorname{deg} v}(v)$ be the number of vertices in each of the components of $T-v$. Then

$$
W(T)=\left(\begin{array}{c}
|V|+1 \\
3
\end{array}\right)-\sum_{v \in V} \sum_{1 \leq i<j<k \leq \operatorname{deg} v} n_{i}(v) n_{j}(v) n_{k}(v) .
$$

Note that this theorem immediately gives the Wiener number of the path $P_{n}$ as in Theorem 1.2, number 5 , as well as the fact that $P_{n}$ has the largest Wiener number of any tree. It would be nice to get analogous results for Wiener polynomials, possibly be comparing them coefficient-wise.

(VI) Because of the development of parallel architectures for interconnection computer networks, there has recently been interest in a generalization of the 
distance concept. A container, $C(u, v)$, is a set of vertex-disjoint paths between two vertices $u, v \in V(G)$, i.e., any two paths in $C(u, v)$ only intersect at $u$ and $v$. The width, $w=w(C(u, v))$, is the number of paths in the container while the length, $l=l(C(u, v))$, is the length of the longest path in $C(u, v)$. For fixed $w$, define the $w$-distance between $u$ and $v$ as

$$
d_{w}(u, v)=\min _{C(u, v)} l(C(u, v)) .
$$

where the minimum is taken over all containers $C(u, v)$ of width $w$. Note that when $w=1$ then $d_{w}(u, v)$ reduces to the usual distance between $u$ and $v$. For more information about these concepts and their relation to networks, see the article of Hsu [8].

Now we can define the $w$-Wiener polynomial by

$$
W_{w}(G ; q)=\sum_{u, v} q^{d_{w}(u, v)}
$$

It would be interesting to compute this polynomial for various graphs and study its properties, e.g., unimodality. It would also be interesting to see if this object yields any useful information in chemistry, group theory, or computer science.

\section{References}

[1] H. Barcelo and A. Goupil, Non broken circuits of reflection groups and their factorization in $D_{n}$, Israel J. Math. 91 (1995), 285-306.

[2] F. Buckley and F. Harary, "Distance in Graphs," Addison-Wesley, Redwood, CA, 1990.

[3] G. Chartrand and L. Lesniak, "Graphs and Digraphs," second edition, Wadsworth \& Brooks/Cole, Monteray, CA, 1986.

[4] I. Gutman, A new method for the calculation of the Wiener number of acyclic molecular graphs, Journal of Molecular Structure (Theochem) 285 (1993), $137-142$.

[5] I. Gutman and O. Polansky, Mathematical Concepts in Organic Chemistry, Springer-Verlag, Berlin, Germany, 1986.

[6] I. Gutman, Y.-N. Yeh, S.-L. Lee J.-C. Chen, Wiener numbers of dendrimers, Comm. Math. Chem. 30 (1994), 103-115. 
[7] F. Harary, "Graph Theory," Addison-Wesley, Reading, MA, 1971.

[8] D. F. Hsu, On container width and length in graphs, groups and networks, IEICE Trans. on Fundamentals of Electronics, Communications and Computer Science, Vol. E77-A, No. 4 (1994), 668-680.

[9] J. E. Humphreys, "Reflection Groups and Coxeter Groups," Cambridge Studies in Advanced Mathematics, Cambridge University Press, Cambridge, 1990.

[10] P. Orlik and H. Terao, "Arrangements of Hyperplanes," Grundlehren 300, Springer-Verlag, New York, NY, 1992.

[11] R. P. Stanley, Log-concave and unimodal sequences in algebra, combinatorics, and geometry, in "Graph Theory and Its Applications: East and West," Ann. NY Acad. Sci. 576 (1989), 500-535.

[12] H. Wiener, Structural determination of paraffin boiling points, J. Amer. Chem. Soc. 69 (1947), 17-20. 